\title{
Formulation Optimization of Polyox Based Modified Release Drug Delivery System
}

\author{
Shah Sunil, Shukla Dilip*, Pandey Harish \\ Sri Satya Sai University of Technology \& Medical Sciences, Sehore (M.P), India-466001
}

\begin{abstract}
Polyethylene Oxide (PEOs) offer specific advantages to be used in pharmaceutical products as release modifiers by forming a hydrogel around the dosage form in aqueous environment causing the drug to follow a diffusional path before releasing into the media. The strength of the hydrogel governs the release retardation capacity of the PEO system. The objective of this dissertation work was to use the Design of Experiments methodology to develop and optimize a PEO based modified release formulation of a highly water-soluble drug like Metoprolol succinate. The effect of the different viscosity grade PEOs, their concentration with respect to the drug, combination of two different viscosity grades, \% drug content in the formulation and the use of water soluble / insoluble fillers on the dissolution of metoprolol succinate was studied. The critical formulation parameters namely PEO concentration and \% drug content were chosen as input factors and dis solution at 1 , 4, 8 and 20 hours was recorded as responses to carry out optimization using the DOE approach. The results obtained after statistical treatment of data provided a design space that can be used for achieving the desired formulation profile. The model has been validated to predict the effect of the input factors (PEO type and concentration and \% drug content) on the responses (in vitro dissolution).
\end{abstract}

Keywords: Polyethylene Oxide, Modified Release Drug Delivery System, Metoprolol

Article Info: Received 19 June 2019; $\quad$ Review Completed 26 July 2019; $\quad$ Accepted 06 Aug 2019; Available online 19 August 2019

\section{Cite this article as:}

Shah S, Shukla D, Pandey H, Formulation Optimization of Polyox Based Modified Release Drug Delivery System, Journal of Drug Delivery and Therapeutics. 2019; 9(4-s):551-561 http://dx.doi.org/10.22270/jddt.v9i4-s.3383

\section{INTRODUCTION}

Modified drug delivery is a domain which requires high degree of skill and understanding in order to regulate the release of the pharmaceutical compound. More often than not, the therapeutic dosing requirement governs whether a particular compound has to be given as a modified drug delivery or as a conventional immediate release dosage form. There can be numerous reasons due to which, the rate of release of the drug from the dosage form needs to be controlled; Primary being to have the drug into systemic circulation at a constant rate for a prolonged period of time so that it elicits a continuous pharmaceutical activity. The drugs with shorter half life, higher doses with narrow therapeutic window, are ideal candidates for delivery as modified release systems. In order to formulate a modified release delivery system, the right combination of scientific principles can in turn help effect the desired kind of release of the drug from the dosage form and thus deliver the drug at the rate and amount as desired or as most beneficial to the body1-3. Based on the mechanism of drug release from the modified release drug delivery systems, they can be classified as Diffusion controlled (matrix and reservoir type ISSN: 2250-1177 of systems) Dissolution controlled, (surface eroding, surface swelling type of systems), Osmotic drug delivery, Multi particulate systems, Enteric coated $(\mathrm{pH}$ dependent systems). Generally, the mechanism of drug release from any kind of modified release delivery system is governed by either of the above mechanisms. The regulation of drug release is achieved by incorporating release retarding agents into the formulations. The most widely used release retarding agents are polymers like high viscosity HPMC for diffusion and dissolution-controlled systems, or $\mathrm{pH}$ sensitive polymers like Eudragits and Ethyl cellulose for enteric coated and Multi particulate systems. Osmotic drug delivery systems involve controlling the release by incorporating an osmogen and a semi permeable membrane into the formulation in order to have a zero order continuous release from the medication ${ }^{4-6}$.

The comparatively less explored approach for regulation of the release from the formulation is the use of Polyoxy ethylene oxides (PEOs) resins. These are available under the brand name of Polyox® and are free flowing and directly compressible powders that form a Hydrogel around the formulation when contact with aqueous environment

CODEN (USA): JDDTAO 
and help in retarding the release of the API from the formulation. They are available in various different viscosity grades based on their molecular weights. They are relatively easy to process into dosage forms because of their free flowing and directly compressible nature and can be used as mere fillers in the tablets in order to act as release retardants. Thus, using PEOs in the formulation does not involve complex set of criticial process variables as observed in case of preparing a matrix, reservoir or a MUPS based delivery system ${ }^{7-9}$. The purpose of this Research work would be to apply design of experiments (DOE) approach to development and optimization of a Polyox based modified release drug delivery system of a highly water-soluble drug. In order to evaluate the effect of Polyox on the release modulation of the drug in the formulation, a model drug was chosen by screening a variety of drug molecules to meet certain pre-defined criteria as mentioned below

$>$ High solubility and high permeability i.e. BCS class I drug. (i.e absorption is not solubility or permeability dependent).

$>$ Biological half life (t1/2) between 2-6 hours to avoid accumulation in the body. Shorter $\mathrm{t} 1 / 2$ will ensure rapid clearance after absorption, thus negating any dose related side effects.

Moderate dose of between 50-150 mg per unit.

$>$ Drug for which use of extensive and elaborative coating process may not be a feasible approach.

Based on the above criteria, Metoprolol succinate was selected as a model drug to evaluate and optimize the formulation related variables associated with polyethylene oxide.

\section{MATERIALS AND METHODS}

Materials: The Active pharmaceutical ingredient Metoprolol succinate used for the study was manufactured by Ipca Laboratories Ltd. Polyethelene oxide polymers were obtained as gift samples from Alpa laboratories ltd India. The other ingredients Prosolv HD 90, directly compressible lactose (Supertab 21), and magnesium stearate were purchased from sources JRS Pharma, Fontenna Excipients and Signet Pharma respectively by Ipca laboratories ltd.

Process of preparation of experimental formulations: The process of preparation of the formulations involved dispensing of raw materials and active, followed by cosifting, mixing and then compression into tablets. A brief step wise description of the manufacturing process is as mentioned below.

Procedure: Dispense the drug substance as per batch requirement. Sift the drug substance through a \# 50 sieve. Dispense the excipients (PEO, Prosolv HD 90 or DCL 21) as per batch requirement.

Co-Sift the excipients along with API through \# 50 sieve 2 -3 times. Load the blend into the blender and Mix blend for 10 min. Add required quantity of magnesium stearate (sifted through \# 50 sieve) and blend for another $5 \mathrm{~min}$. Compress tablets equivalent to make $100 \mathrm{mg}$ Metoprolol succinate. A flow chart of the manufacturing procedure is depicted below.



\section{Figure 1: Flow chart for manufacturing of Metoprolol succinate PEO tablets}

Analytical methodology: The analytical methodology for in vitro drug dissolution was adapted from the Official USP monograph for Metorpolol succinate extended release tablets. The tablets were analyzed for content of metoprolol succinate in the dissolution medium. The details of the method are as mentioned below

In vitro Dissolution method: The in vitro dissolution analysis was carried out in $\mathrm{pH} 6.8$ phosphate buffer, $500 \mathrm{ml}$ in USP type II paddle Apparatus at $50 \mathrm{rpm}$. The samples were withdrawn using auto sampler at 1, 4, 8, 12, 20 hours. The volume of samples withdrawn was replaced by additional media from replacement bowl.

HPLC analysis: The HPLC analysis was carried out using a 4 mm X $12.5 \mathrm{~cm}$ column containing C18 packing. The mobile phase of pH 3.0 phosphate buffer: Acetonitrile (375:125) was used for elution at a flow rate of $1 \mathrm{ml} / \mathrm{min}$. The wavelength of absorption was determined using a PDA detector in the HPLC where Metoprolol succinate showed two maxima at wavelengths of $222 \mathrm{~nm}$ and $275 \mathrm{~nm}$. The $222 \mathrm{~nm}$ maxima showed a greater response and hence it was chosen as the wavelength for estimation for all the dissolution samples.

Preparation of $\mathrm{pH} 3$ Phosphate buffer: Mixed 50ml of 1M monobasic sodium phosphate and $8.0 \mathrm{ml}$ of $1 \mathrm{M}$ phosphoric acid and diluted with water to $1000 \mathrm{ml}$. Adjusted $\mathrm{pH}$ to 3.0 with $1 \mathrm{M}$ monobasic potassium phosphate or $1 \mathrm{M}$ phosphoric acid.

Preparation of standard solution: Dissolved $50 \mathrm{mg}$ accurately weighed Metoprolol succinate in Mobile Phase to obtain a solution of known concentration of about $0.05 \mathrm{mg}$ per $\mathrm{ml}$ (i.e. $50 \mathrm{ppm}$ ). $20 \mu \mathrm{l}$ of injection volume was used to determine the response.

Preparation of sample solution: The $2 \mathrm{ml}$ aliquot from the samples collected in at each time point in dissolution vial and diluted with the dissolution medium to $5 \mathrm{ml}$ in a volumetric flask. Injected $20 \mu \mathrm{l}$ of sample solution in the HPLC column. The standard curve was prepared to determine the linearity of the response area (area under the peak) across different concentrations and the R2 value was found to be 0.998 . The figure 2 below depicts the standard linearity graph.

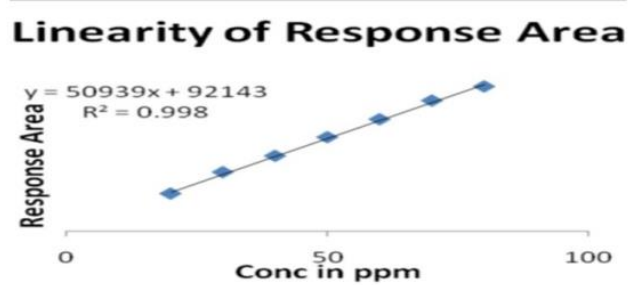

Figure 2: Standard Response linearity with respect to concentration 


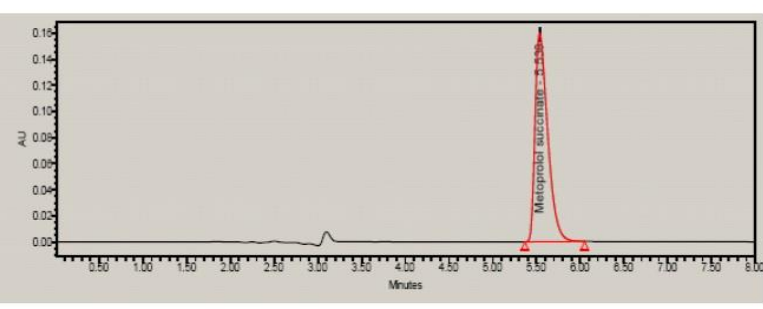

Chromatogram of $50 \mathrm{ppm}$ Standard

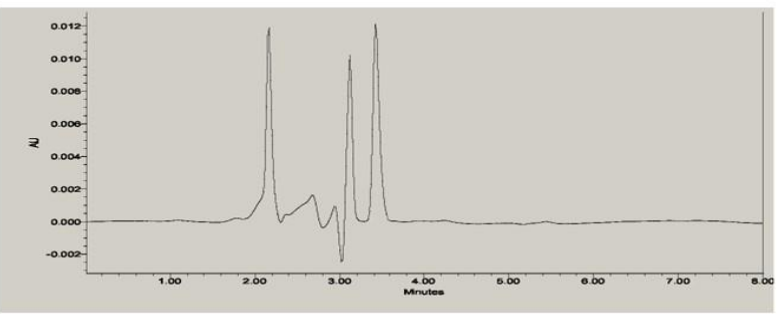

Chromatogram of blank

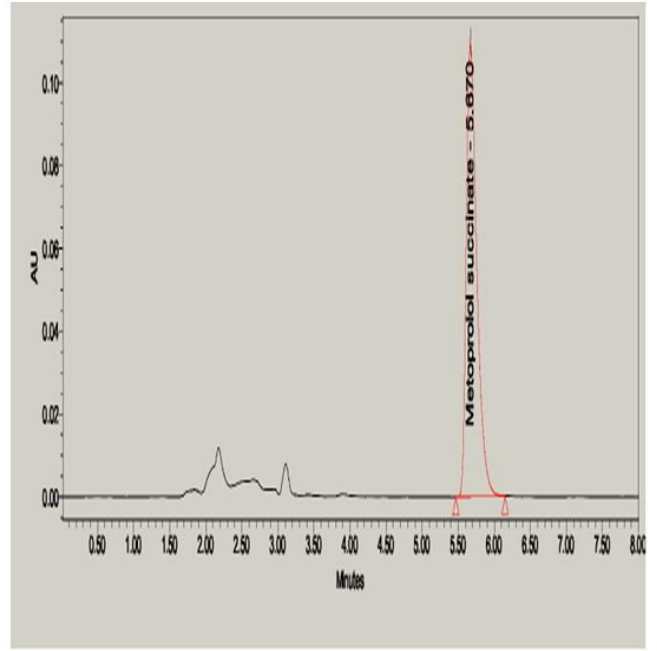

Chromatogram of sample

Figure 3: Chromatograms of the standard, blank and sample

\section{RESULTS AND DISCUSSION}

The results of the experimental formulations were compiled in the DOE response sheet in the Design expert $\AA$ software screen as mentioned in the Figure 4 below and the further subjected to data treatment to arrive at statistically significant factors that impact the formulation design. The $12^{\text {th }}$ hour dissolution was performed only to arrive at profile across the time range but this response was not used for data analysis and optimization in the DOE.

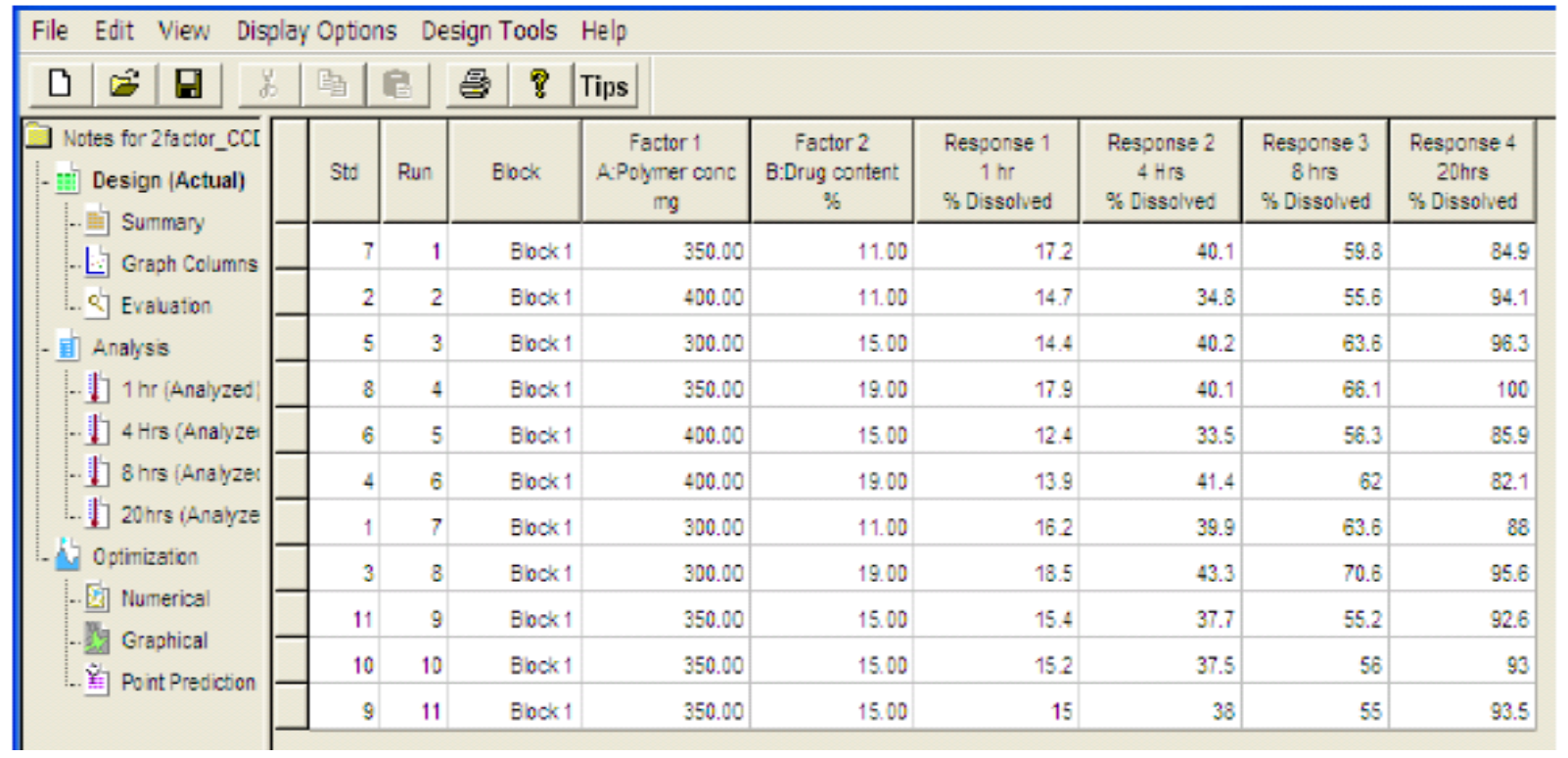

Figure 4: DOE Experimental design and responses

The Quadratic model was chosen for fitting the data as it is the most preferred model for fitting the CCD design and no aliases were found in the model. Since the model chosen is quadratic, a second order polynomial would be used for predicting the response functions.

$$
Y=\beta 0+\beta 1 A+\beta 2 B+\beta 3 A^{2}+\beta 4 B^{2}+\beta 5 A B
$$

Where, $\mathrm{Y}$ is the response, $\mathrm{A}$ and $\mathrm{B}$ are independent variables and $\beta 0$ to $\beta 5$ are the constants or coefficients for each term in the equation. The responses desired in the study were dissolution at time points equivalent to $1,4,8$ and 20 hours and the table 1 below depicts the low and high values for the variables (as chosen in the study) and responses (as achieved by experimentation). It can be observed that the ratio of maximum to minimum in case of all responses is less than 2.0. Hence the data does not require any transformation. 
Table 1: Response compilation in DOE

\begin{tabular}{|l|c|c|c|c|c|c|c|}
\hline \multicolumn{1}{|c|}{ Name } & Units & Type & Desired & Low & High & Std. Dev & Ratio of Max To \\
\hline Polymer conc & mg & Factor & - & 300 & 400 & NA & NA \\
\hline Drug content & \% & Factor & - & 11 & 19 & NA & NA \\
\hline $1 \mathrm{hrs}$ & \% Dissolved & Response & $<25 \%$ & 12.4 & 19 & 0.3006 & 1.492 \\
\hline $4 \mathrm{hrs}$ & \% Dissolved & Response & $20-40 \%$ & 33.5 & 43.3 & 2.0381 & 1.293 \\
\hline $8 \mathrm{hrs}$ & \% Dissolved & Response & $40-60 \%$ & 55 & 70.6 & 1.6746 & 1.284 \\
\hline $20 \mathrm{hrs}$ & \% Dissolved & Response & $80-100 \%$ & 82.1 & 100 & 4.3736 & 1.218 \\
\hline
\end{tabular}

The results obtained for each of the responses are mentioned in the table 2 . The selected model for each response is underlined.

Table 2: Sequential Model sum of Squares

\begin{tabular}{|c|c|c|c|}
\hline Response & Model & F Value & P value \\
\hline \multirow{4}{*}{ D @ 1 hour } & Linear & 2.26 & 0.1665 \\
\cline { 2 - 4 } & 2FI & 0.92 & 0.3704 \\
\cline { 2 - 4 } & Quadratic & 99.06 & 6.001 \\
\cline { 2 - 4 } & Cubic & 0.18 & 0.02 \\
\hline \multirow{4}{*}{ D @ 4 hours } & Linear & 5.77 & 0.4696 \\
\cline { 2 - 4 } & 2FI & 0.58 & 0.0983 \\
\cline { 2 - 4 } & Quadratic & 3.82 & 0.0055 \\
\cline { 2 - 4 } & Cubic & $46.37 *$ & 0.0309 \\
\hline \multirow{4}{*}{ D @ 8 hours } & Linear & 5.54 & 0.9432 \\
\cline { 2 - 4 } & 2FI & 5.46 & 0.0052 \\
\cline { 2 - 4 } & Quadratic & 18.06 & 0.9589 \\
\hline \multirow{4}{*}{ D @ 20 hours } & Cubic & 0.0043 & 0.3368 \\
\cline { 2 - 4 } & Linear & 1.25 & 0.0600 \\
\cline { 2 - 4 } & 2FI & 5.02 & 0.6886 \\
\cline { 2 - 4 } & Quadratic & 0.40 & 0.0004 \\
\hline
\end{tabular}

* cubic model is aliased for a central composite design. Hence design augmentation required to remove the alianses and estimate the higher order terms. Hence here, the next best $\mathrm{F}$ value model is selected for evaluation.

The complete ANOVA output for all the responses is described later in table 3, 4 and 5 . Here also a higher $\mathrm{F}$ value and $\mathrm{p}$ value less than 0.1 (preferably < 0.05 ) indicates a significant model chosen for the data.

Table 3: ANOVA output for response surface - Model

\begin{tabular}{|c|c|c|c|c|c|}
\hline Response & Model & Model F & P value & Significant & Final equation in \\
\hline D-1 hour & Quadratic & 70.92 & 0.001 & $\mathrm{~A}, \mathrm{~B}, \mathrm{AB}, \mathrm{A} 2$, & $+15.18-1.35^{*} \mathrm{~A}+0.37^{*} \mathrm{~B}-0.78^{*} \mathrm{~A}$ \\
\hline D-4 hours & Linear & 5.77 & $0.02 \ldots 81$ & $\mathrm{~A}, \mathrm{~B}$ & $+38.77-2.28^{*}$ \\
\hline D-8 hours & Quadratic & 14.51 & 0.0031 & $\mathrm{~A}, \mathrm{~B}, \mathrm{~B} 2$ & $+57.22-3.98^{*} \mathrm{~A}$ \\
\hline D-20 hours & 2 FI & 2.93 & 0.109 & $\mathrm{~A}, \mathrm{~B}$ & $+91.45-2.97 * \mathrm{~A}+$ \\
\hline
\end{tabular}

If extra design points beyond what's needed for the model are added, and some points were replicated to provide an estimate of pure error, the results of a Lack of Fit test for each model also can be calculated. Lack of Fit compares the residual error (MEAN SQUARE) to the pure error (MEAN
SQUARE). Lack of fit is NOT desirable so a small $\mathrm{F}$ value and $\mathrm{p}$ value greater than 0.05 (preferably $<0.1$ ) are desired. If a model shows significant lack of fit, it should not be used to predict the response. The lack of fit calculation for the four responses is described in the table 4 below.

Table 4: Model Lack of Fit calculation for four responses

\begin{tabular}{|c|c|c|c|c|}
\hline Response & Model Chosen & F Value & P value & Character \\
\hline D @ 1 hour & Quadratic & 3.10 & 0.2534 & In-significant \\
\hline D @ 4 hours & Linear & 87.12 & 0.1114 & Marginally Significant \\
\hline D @ 8 hours & Quadratic & 16.03 & 0.0593 & In- significant \\
\hline D @ 20 hours & 2FI & 131.30 & 0.0076 & Significant \\
\hline
\end{tabular}

The other objective can be where the primary concern is to simply identify factors and interactions that are affecting the response and generally just learn if higher or lower factor levels are better (generally with factorial designs). In this case, there might have the situation where the model is statistically significant and there is no lack of fit, but the Rsquared's are low. It can be concluded that the significant terms identified are correct and the graphs will show the ISSN: 2250-1177 best directions to follow, but all the sources of variation are not found (and hence not controlled) in the formulation or process. There are other things left unidentified which may or may not also give even better results. So, there is no doubt that the factors found are correct and their model is adequate, but there is more to be investigated. When the Rsquared values are low then the model should not be used for prediction as it doesn't explain enough of what is going CODEN (USA): JDDTAO 
on in totality. A good next step would be to set the known factors at their best settings and then brain-storm about other possible factors and run another DOE. The R square values for all four responses are compiled in the table 5 below.

Table 5 : R2 Values for all four responses

\begin{tabular}{|c|c|c|c|c|c|}
\hline Response & R2 & R2 Adjusted & R2 Predicted & Adequate precision & PRESS \\
\hline D @ 1 hour & 0.9861 & 0.9722 & 0.8745 & 28.065 & 4.08 \\
\hline D @ 4 hours & 0.5906 & 0.4883 & 0.1117 & 7.442 & 71.63 \\
\hline D @ 8 hours & 0.9861 & 0.9043 & 0.8439 & 12.225 & 56.26 \\
\hline D @ 20 hours & 0.5564 & 0.3332 & -0.9814 & 5.996 & 598.05 \\
\hline
\end{tabular}

As is described in the table above, the cubic model showed better F values fo the D@4 hours and D@20 hours indicating higher order terms impacting the responses. Since the D@20 hours response is the final time point in the dissolution and the specification of Dissolution $>80 \%$ meets for all points across the design points this response need not be used for predictive purposes. Also, the low predictability observed for D@4 hours can be overlooked as it has a brackting of D@1 hour and D@8 hours which in turn can be predicted acturately from the model. But a final full proof solution to better fitting of data to all responses would be to augment the design to better estimate higher order terms to fit the cubic model to these two responses. The current work limits to identification of the significant factors for responses D@4 hours and D@20 hours and ascertains that the predictive power of the model is low for these responses.

The normal probability The Normal probability plots for the four responses are plotted in figure $5 \& 6$ depict that the distribution is uniform and there is no specific pattern in the distribution.

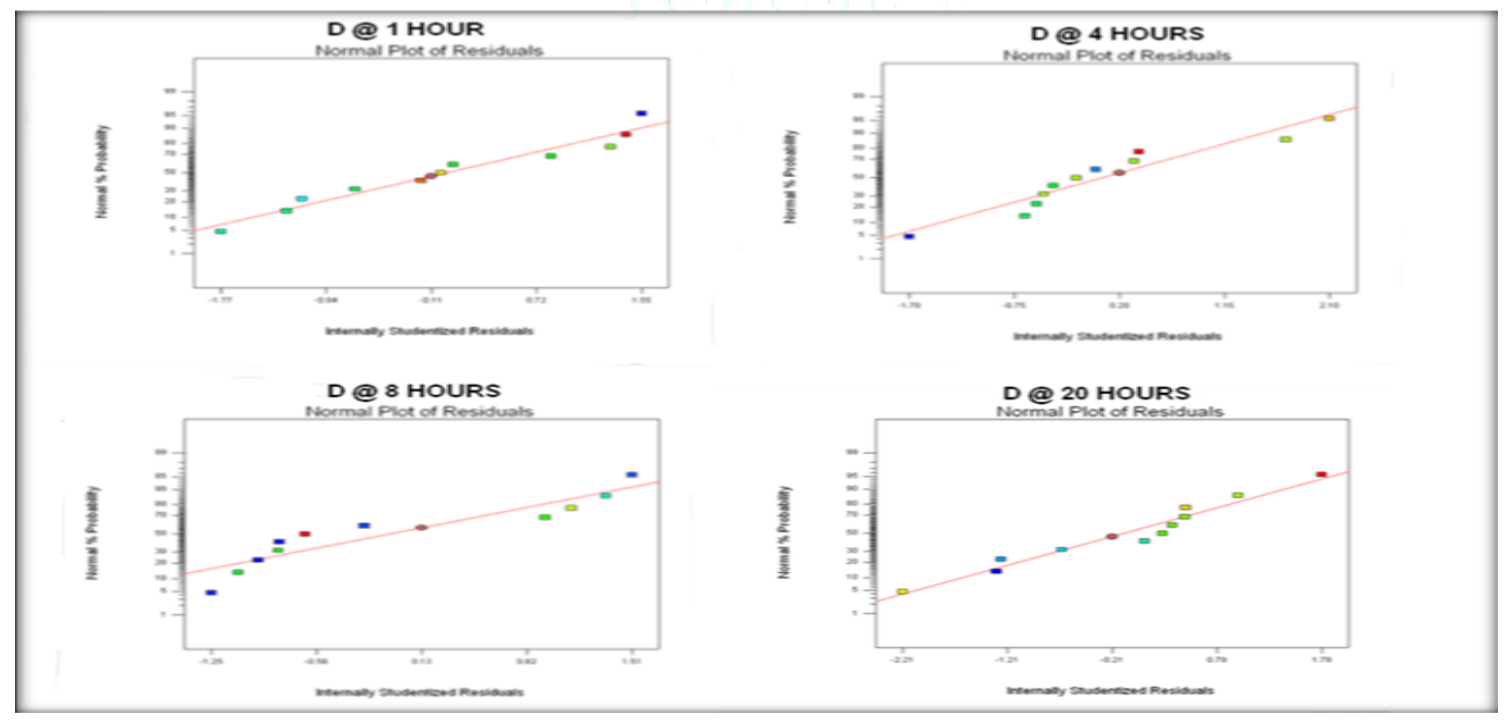

Figure 5: Normal probability plots

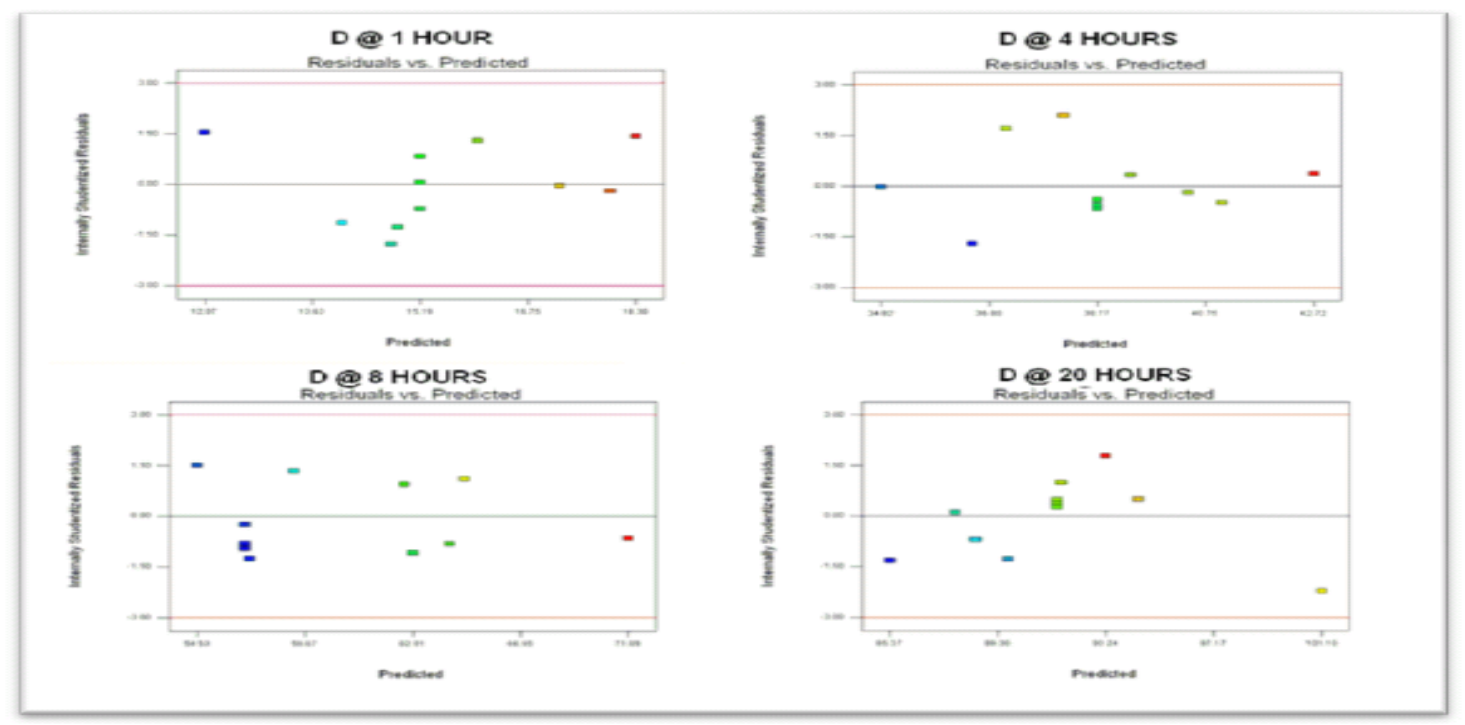

Figure 6: Residuals vs Predicted 
The DOE software analyses the differences between the actual values obtained for experimental runs and the predicted values one would have arrived at if the models were used to predict the response at the set of factor combinations corresponding to the runs. The details for each of the responses are compiled in the table 6 and 7 .

Table 6: Predicted vs Actual Compilation - Response D@ 1hour and D@ 4 hours

\begin{tabular}{|c|c|c|c|c|c|c|}
\hline \multirow{2}{*}{$\underset{\text { Order }}{\text { Standard }}$} & \multicolumn{3}{|c|}{ D @ 1 Hour } & \multicolumn{3}{|c|}{ D @ 4 Hours } \\
\hline & $\begin{array}{c}\text { Actual } \\
\text { value }\end{array}$ & $\begin{array}{c}\text { Predicted } \\
\text { value }\end{array}$ & Residual & $\begin{array}{l}\text { Actual } \\
\text { value }\end{array}$ & $\begin{array}{l}\text { Predicted } \\
\text { Value }\end{array}$ & Residual \\
\hline 1 & 16.2 & 16.0214 & 0.178 & 39.9 & 39.389 & 0.510 \\
\hline 2 & 14.7 & 14.8714 & -0.171 & 34.8 & 34.822 & -0.022 \\
\hline 3 & 18.5 & 18.3048 & 0.195 & 43.3 & 42.722 & 0.577 \\
\hline 4 & 13.9 & 14.0548 & -0.154 & 41.4 & 38.156 & 3.243 \\
\hline 5 & 14.4 & 14.7736 & -0.373 & 40.2 & 41.056 & -0.856 \\
\hline 6 & 12.4 & 12.0736 & 0.326 & 33.5 & 36.489 & -2.989 \\
\hline 7 & 17.2 & 17.2070 & -0.007 & 40.1 & 37.106 & 2.993 \\
\hline 8 & 17.9 & 17.9403 & -0.0403 & 40.1 & 40.439 & -0.339 \\
\hline 9 & 15 & 15.1842 & -0.184 & 38 & 38.772 & -0.772 \\
\hline 10 & 15.2 & 15.1842 & 0.0157 & 37.5 & 38.772 & -1.272 \\
\hline 11 & 15.4 & 15.1842 & 0.215 & 37.7 & 38.772 & -1.072 \\
\hline
\end{tabular}

Table 7: Predicted vs Actual Compilation - Response D@ 8 hour and D@ 20 hours

\begin{tabular}{|c|c|c|c|c|c|c|}
\hline \multirow{2}{*}{$\begin{array}{c}\text { Standard } \\
\text { Order }\end{array}$} & \multicolumn{3}{|c|}{ D @ 8 Hours } & \multicolumn{3}{c|}{ D @ 20 Hours } \\
\cline { 2 - 7 } & Actual & Predicted & Residual & Actual & Predicted & Residual \\
\hline 1 & 63.6 & 63.5 & 0.100 & 88 & 90.08 & -2.082 \\
\hline 2 & 55.6 & 55.833 & -0.233 & 94.1 & 93.94 & 0.152 \\
\hline 3 & 70.6 & 70.366 & 0.233 & 95.6 & 100.41 & -4.815 \\
\hline 4 & 62 & 62.1 & -0.100 & 82.1 & 84.68 & -2.582 \\
\hline 5 & 63.6 & 61.203 & 2.397 & 96.3 & 95.248 & 1.052 \\
\hline 6 & 56.3 & 53.236 & 3.063 & 85.9 & 89.31 & -3.415 \\
\hline 7 & 59.8 & 59.666 & 0.133 & 94 & 92.015 & 1.985 \\
\hline 8 & 66.1 & 66.233 & -0.133 & 100 & 92.548 & 7.452 \\
\hline 9 & 55 & 57.22 & -2.220 & 93.5 & 92.28 & 1.218 \\
\hline 10 & 56 & 57.22 & -1.220 & 93 & 92.28 & 0.718 \\
\hline 11 & 55.2 & 57.22 & -2.020 & 92.6 & 92.28 & 0.318 \\
\hline
\end{tabular}

The residual values in the tables above indicate that there is no significant variation in the actual responses obtained and those predicted by the model. The Predicted Vs Actual plots for all responses are depicted in the figure 7 . It can be observed that the predicted vs actual point are more closer to the line for responses D@ 1 hour and D @ 8 hours indicating better fit of model to the data. The points are more scattered and away from the line for the D @ 4 hours and D @ 20 hours reponses reconfirming that the models chosen is not a perfect' fit for predicting the data for these two responses. As indicated above, the augmentation of design is a better alternative to further fit these responses to a higher order model. 


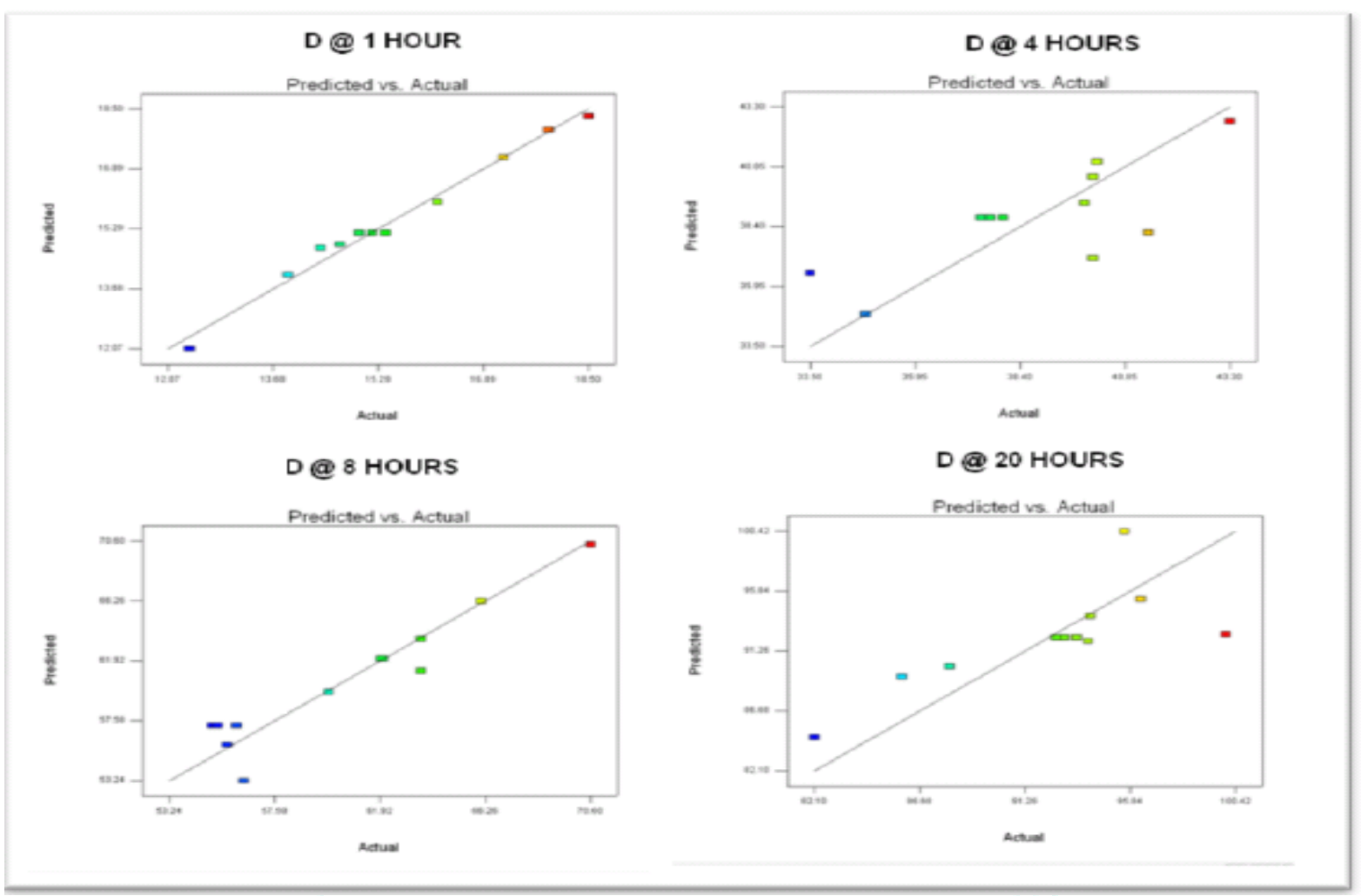

Figure 7: Predicted vs Actual Plots

The perturbation plot helps to compare the effect of all the factors at a particular point in the design space. The response is plotted by changing only one factor over its range while holding of the other factors constant. By default, Design-Expert software sets the reference point at the midpoint (coded 0 ) of all the factors. But it can be changed to any point (perhaps the optimal run conditions) by using other Factors Tool in the software. A steep slope or curvature in a factor shows that the response is sensitive to that factor. A relatively flat line shows insensitivity to change in that particular factor. If there are more than two factors, the perturbation plot could be used to find those factors that most affect the response. These influential factors are good choices for the axes on the contour plots. This plot is like "one factor at a time" experimentation - it does not show you the effects of interactions. Since this study was carried out with only two factors, the perturbation plots were used to only study the effect of factors on the responses and not to narrow down the influential factors for the contour plots. The figure 8 depicts the perturbation plots for all the four responses while keeping the factors at midpoint levels.

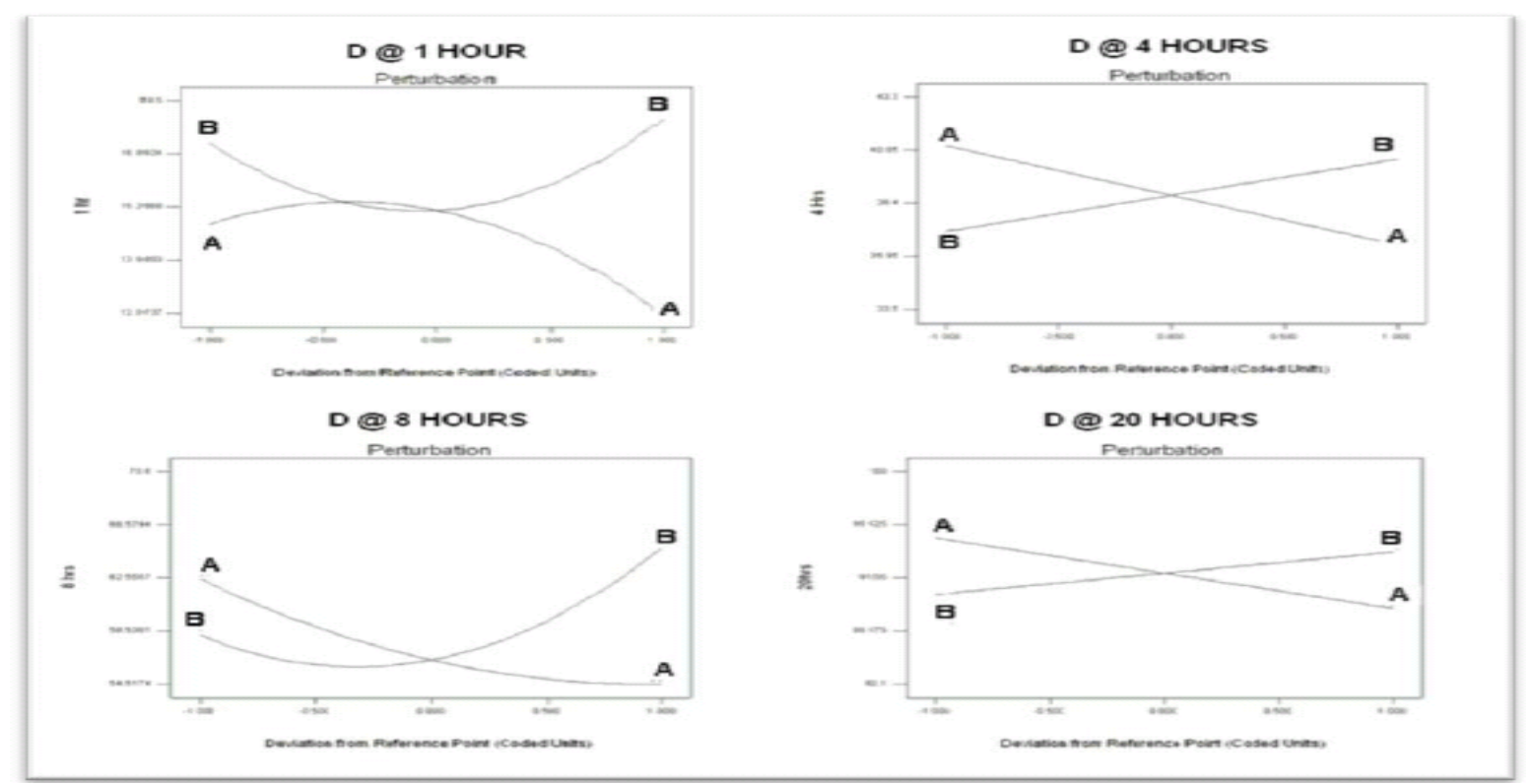

Figure 8: Perturbation Plots 
These perturbation plots show that all the responses are sensitive to the factors as in all cases there is a visible steep slope or curvature in the response plots. After evaluating the perturbation, the factors can now be used to generate 2D contour plots and 3D response surface plots. It can also be reconfirmed from the perturbation plots that D@1 hour and D@8 hours are quadratic responses as they show a curve wheres D@4 hours and D@20 hours show a straight line indicating linearity (in given experimental data). Also a greater curvature and significant slope for the linear response indicate that both factors have impact on each of the responses. The Contour plots for the four responses are plotted in the figures. Each line on the contour represents the same responses at the combination of the two factor levels.

It can be seen that at higher polymer concentrations, and around 13 to $17 \%$ of drug content can help achieve minimum dissolution value at the 1 hour time point. Also, at all of the factor combinations, the dissolution does not go beyond the desired limit of $25 \%$ indicating that the chosen factor levels are competent to achieve the desired response. The corresponding 3D response surface for this response is depicted below for better interpretation and visualization of the response surface. It can be observed that the contours for the D @ 4 hours response are linear. It can also be seen that towards lower polymer

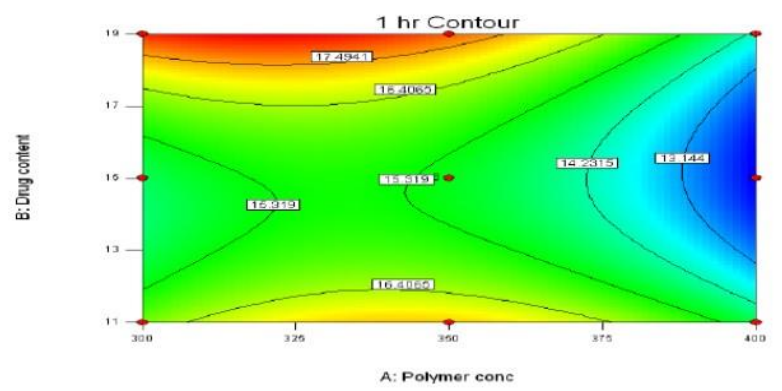

Contour plot for D@1 hour

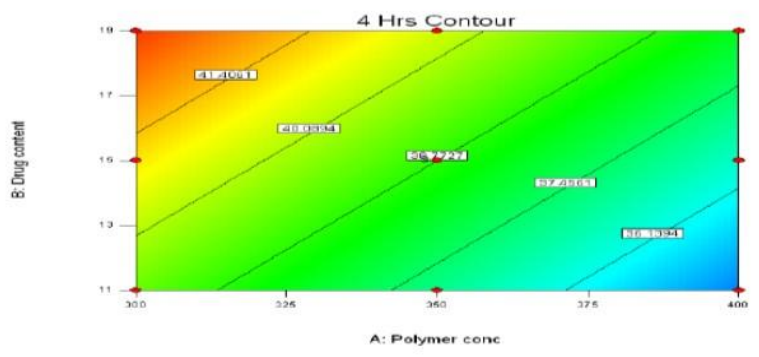

Contour plot D@4hours concentration (less than $325 \mathrm{mg}$ ) and at higher drug \% ( greater than $16 \%$ ), the dissolution value is above $40 \%$ (as against the desired limit of $20-40 \%$ ). Hence to be safely inside the limit, one has to choose a higher polymer concentration (greater than 375mg) and lower drug concentration (between 11 to $13 \%$ ) to have a lower dissolution value as shown by the $36 \%$ contour at bottom right corner in the above figure. The $3 \mathrm{D}$ response surface for this response also helps interpret a linear relationship and the graph is depicted in figure.

The contour plot for the D @ 8 hours response is again a curvature-based plot indicating higher order terms. The contour indicates desired reponse above $350 \mathrm{mg}$ polymer concentration and between 11 to $15 \%$ drug content. In this plot too, at lower polymer concentration and above 15 $\%$ drug content, the dissolution value tends to rise above the spec limit (i.e. $60 \%$ ). The contour and the corresponding 3D response surface for D @ 8 hours response is depicted in figures. Although, the response surface is not a good predictor for the response D @ 20 hours, the contour and 3D response surface were constructed to visualize the effect of factors combinations on to the response. This contour plot does not show any pattern (curvature or linearity) with respect to the responses.

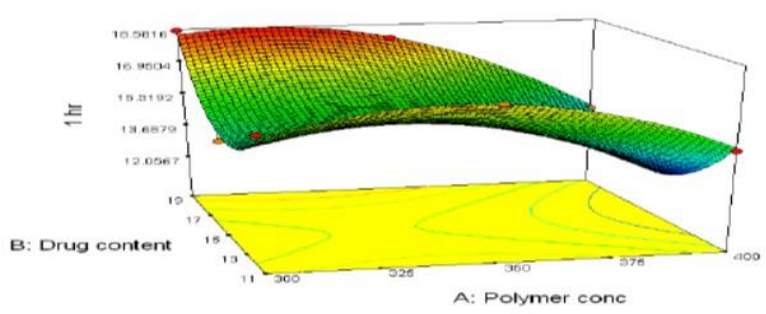

3D Response surface for D @ 1 hour

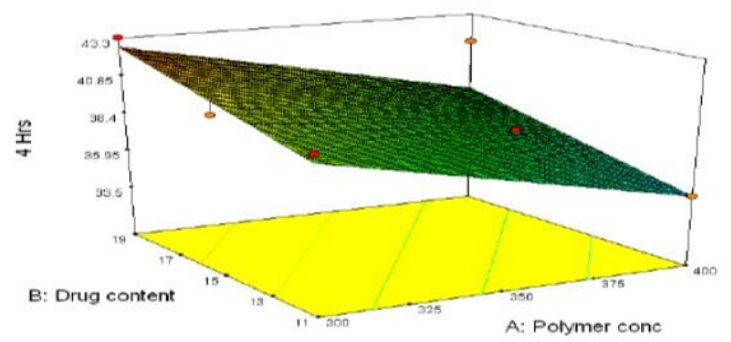

3D Response surface for D @ 4 hours

Figure 9: Contour plot with 3D response in 1 hour and 4 hours 


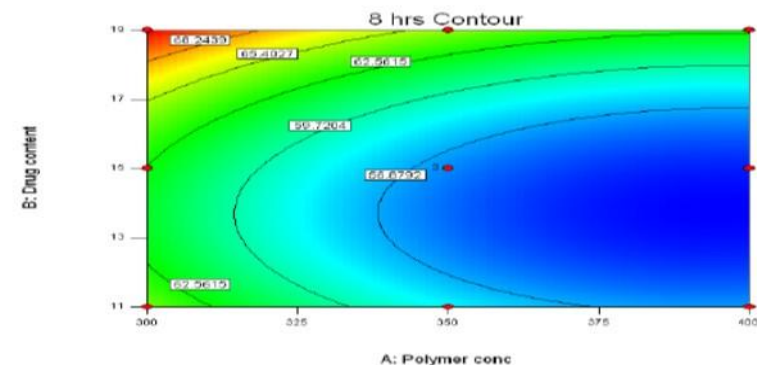

Contour plot for D@8 hours

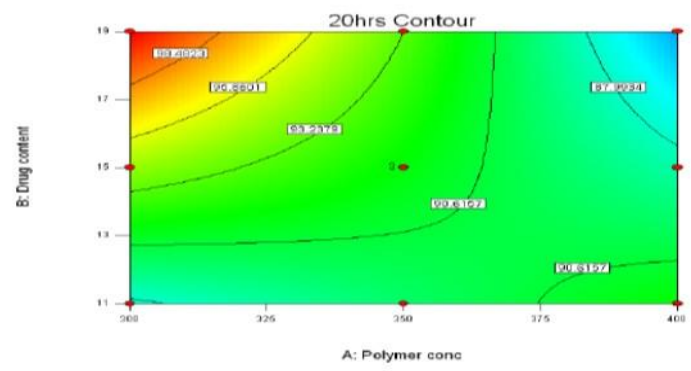

Contour plot D@20 hours

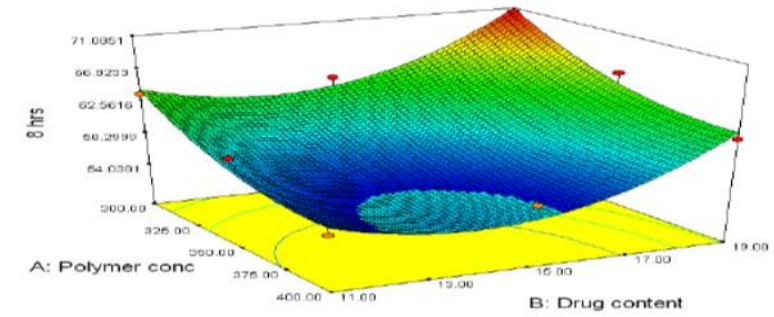

3D Response surface for D @ 8 hours

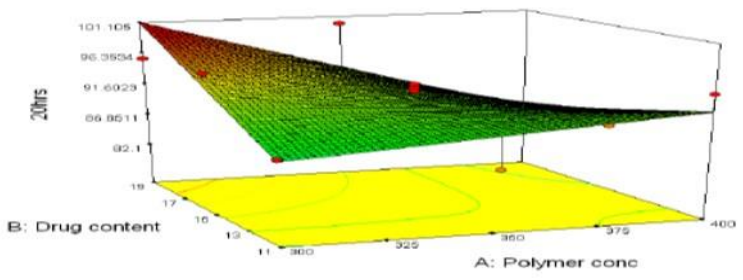

3D Response surface for D@ 20 hours

Figure 10: Contour plot with 3D response in 8 hour and 20 hours

\section{OPTIMIZATION:}

The optimization module in Design-Expert searches for a combination of factor levels that simultaneously satisfy the requirements placed on each of the responses and factors. It can be only be used when each response has been analyzed independently to establish the appropriate model. Optimization of one response or the simultaneous optimization of multiple responses can be performed graphically or numerically. Also, one can simultaneously evaluate all the response models for any value of the independent variables using the point prediction node. Numerical Optimization: The table 8 below compiles the Numerical optimization criteria for each response in order to carry out numerical optimization.

Table 8: Numerical Optimization constraints

\begin{tabular}{|c|c|c|c|c|}
\hline Name & Goal & Lower Limit & Upper Limit & Weight \\
\hline Polymer conc & Is in range & 300 & 400 & 3 \\
\hline Drug content & Is in range & 11 & 19 & 3 \\
\hline $1 \mathrm{hrs}$ & Is in range & 10 & 20 & 3 \\
\hline $4 \mathrm{hrs}$ & Is in range & 30 & 40 & 3 \\
\hline $8 \mathrm{hrs}$ & Is in range & 40 & 60 & 3 \\
\hline $20 \mathrm{hrs}$ & Is in range & 80 & 100 & 3 \\
\hline
\end{tabular}

The figure 11 below shows the screen shot of possible solutions suggested by the software design expert ${ }^{\circledR}$ after considering the desired constraints during numerical optimization of the design.

Graphical Optimization: With multiple responses one needs to find regions where critical properties. By superimposing or overlaying critical response contours on a single plot we can visually search for the best combination. When dealing with many input variables, its better to do numerical optimization first, otherwise its impossible to uncover a feasible region. Graphical optimization displays the area of feasible response values in the factor space. Regions that do not fit the optimization criteria are shaded. For multiple responses we may see several overlapping shaded areas. Any "window" that is NOT shaded satisfies the multiple constraints on the responses. The figure 11 depicts the overlaying contours for each response meeting the criteria set in table 8 above The region (yellow) depicts the design space' for achieving the set of responses with the factor combinations. requirements simultaneously meet the critical properties. By superimposing or overlaying critical response contours on a single plot we can visually search for the best combination. When dealing with many input variables, its better to do numerical optimization first, otherwise its impossible to uncover a feasible region. Graphical optimization displays the area of feasible response values in the factor space. Regions that do not fit the optimization criteria are shaded. For multiple responses we may see several overlapping shaded areas. Any "window" that is NOT shaded satisfies the multiple constraints on the responses. The figure 11 depicts the overlaying contours for each response meeting the criteria set in table 8 above The region (yellow) depicts the design space' for achieving the set of responses with the factor combinations. 


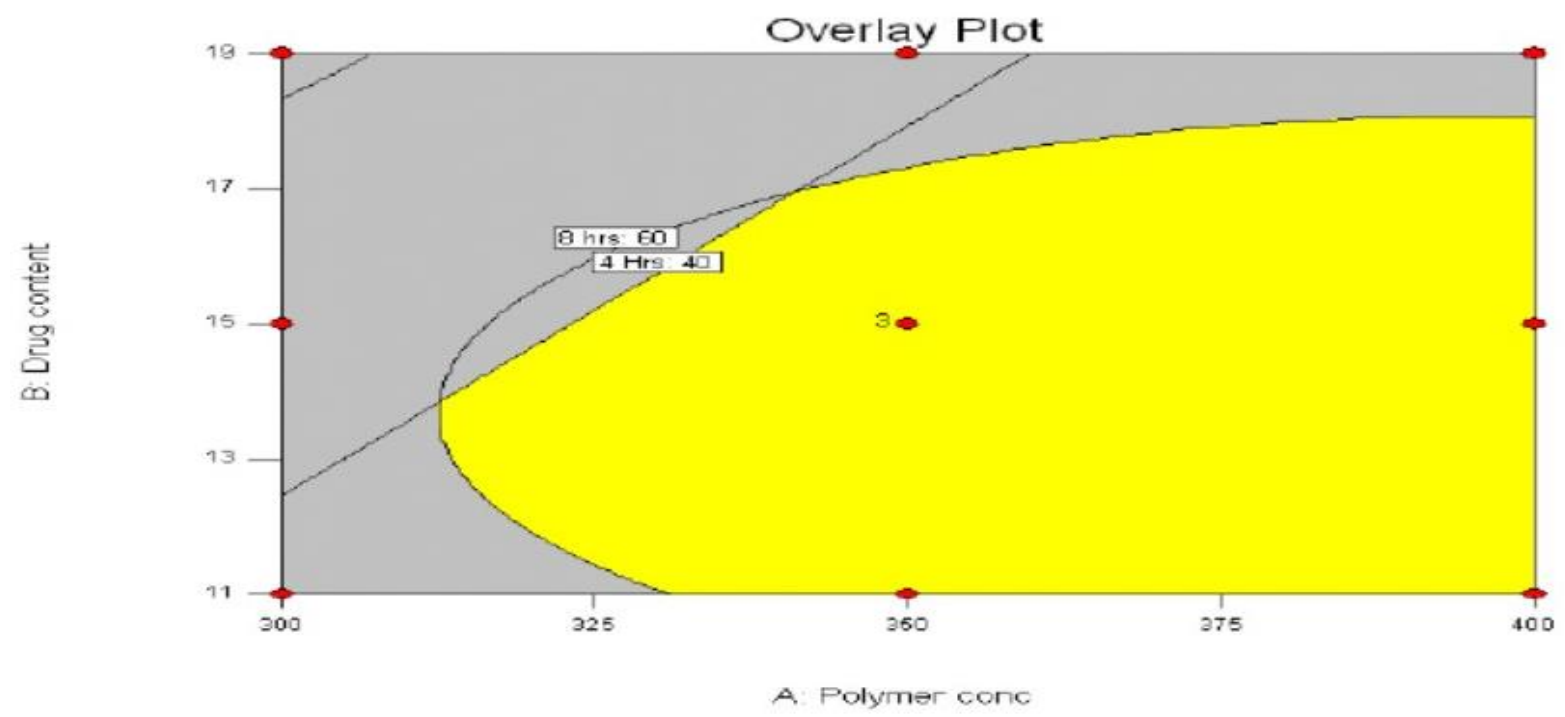

Figure 11: Graphical optimization overlay showing the Design Space

Validation of the model: The validation of the model was carried out using the point prediction' tool in the design expert software. The tool can be used to predict the response by varying the factor combinations. A setting of factors was chosen in the design space and replicated 3 times to determine the error in predictability of the design and to determine the predicted vs actual variation of the model. The table 9 describes the factor combinations chosen and their predicted vs the actual response.

Table 9: Predicted vs Actual responses

\begin{tabular}{|c|c|c|c|c|c|}
\hline Trial & Factor & Response & Predicted value & Actual value & $\begin{array}{c}\text { Residual } \\
\text { (Actual -Predicted) }\end{array}$ \\
\hline \multirow{14}{*}{1} & \multirow{14}{*}{$\begin{array}{l}\text { Polymer } \\
\text { concentration } \\
(350 \mathrm{mg} \text {; Drug Content }) 15 \\
\%\end{array}$} & \multirow{6}{*}{1 hour } & \multirow{6}{*}{15.1959} & 15.3 & - \\
\hline & & & & - & -0.1041 \\
\hline & & & & - & - \\
\hline & & & & 15.2 & -0.0041 \\
\hline & & & & 16 & -0.8041 \\
\hline & & & & 37.5 & 1.3178 \\
\hline & & \multirow[t]{2}{*}{4 hours } & \multirow{2}{*}{38.8178} & 37.8 & 1.0178 \\
\hline & & & & 38.2 & 0.6178 \\
\hline & & \multirow{4}{*}{8 hours } & \multirow{4}{*}{57.3129} & 56.4 & 0.9129 \\
\hline & & & & 57.5 & -0.1871 \\
\hline & & & & 58.4 & -1.0871 \\
\hline & & & & 92 & -0.4973 \\
\hline & & \multirow{2}{*}{20 hours } & \multirow{2}{*}{91.5027} & 91.5 & 0.0027 \\
\hline & & & & 93.5 & -1.9973 \\
\hline
\end{tabular}

It can be seen that the data of predicted vs actual are in reasonable agreement of each other. Thus, the model can be used to navigate the design space successfully.

\section{SUMMARY AND CONCLUSIONS}

The Polyethylene oxide polymers provide a very unique processing advantage over other conventional polymers used in modified release formulation systems. The concentration of the polymer and its grade chosen are the most important factors that affect the formulation design and they have to be chosen based on the desired product performance. The combinations of two viscosity grades do not significantly impact the dissolution of the drug from the system. The use of a high dose highly water-soluble drug presents an added challenge to formulate a modified release dosage form. Initial experiments were carried out to arrive at factors critical to the formulation in order to meet the ISSN: 2250-1177 desired release criteria. From the results obtained in these trials the polymer was chosen to be PEO WSR 303 and its concentration and the \% drug content of the total tablet weight were chosen to be the factors most affecting the formulation performance. A structured optimization study was undertaken using the Design of experiments approach. A Two factor 3 level face centered Central composite design was chosen with polymer concentration and \% drug content being the two factors. The polymer concentration was varied from 300 to $400 \mathrm{mg}$ per tablet and the drug content between 11 to $19 \%$ of the tablet weight. Dissolution at 1, 4, 8- and 20-hours' time points was chosen as the output responses to determine the \% drug release. The experimental runs were executed and the results obtained subjected to data treatment and multivariate analysis. The statistical correlations were drawn based on the predictive and suggestive tools in the DOE choosing the 
statistical models for predicting each of the responses. The Dissolution at 1 hour and 8 hours followed a quadratic model whereas the models chosen for dissolution at 4 hours and dissolution at 20 hours were estimated a linear and $2 \mathrm{FI}$ model respectively. The ANOVA was calculated to determine the significant factors in the model that affect the response outcomes. After fitting of data to the selected models optimization was carried out for achieving the target product profile. The repeatability of the experiments was evaluated by choosing a point in the design space and replicating 3 times to determine the inter response variations and the difference was found to be statistically in-significant. The design of experiments (DOE) approach was thus used to optimize the polyethylene oxide based modified drug delivery system to meet the target product profile for the highly water soluble compound of Metoprolol succinate.

\section{Value addition of this work}

The fundamentals and basics of DOE (Design of experiment) learned during this work can be applied to other projects where meeting the desired target product profiles is highly critical. Although, Metoprolol succinate was used as a model drug, the PEO based platform technology can be applied with minor modifications to other compounds with similar properties in order to meet the target specifications. Developing a PEO based modified release drug delivery system can in turn help circumvent the innovator IP and reduce the formulation and process time involved in development thus saving cost and improving throughput responses.

\section{ACKNOWLEDGEMENT}

Authors are thankful to SSSUTMS, School of Pharmacy, Sehore and Ipca as well as Alpa laboratories ltd Indore, for providing the necessary facilities \& guidance to complete this research.

\section{REFERENCES}

1. Regardh G, Borg KO, Johnsson R, Johnsson G and Palmer L, Pharmacokinetic studies on the selective $\beta 1$-receptor antagonist metoprolol in man, Journal of Pharmacokinetic and Biopharmaceutical, 1974; 2: 347-364.

2. Kendall MJ, Maxwell SR and Westergren SA, Controlled release metoprolol: Clinical pharmacokinetic and therapeutic implications, Clinical Pharmacokinetic, 1991; 5: 319-330.

3. Ragnarsson G, Sandberg A, Jonsson UE and Ggren SJ, Development of A New Controlled Release Metoprolol Product, Drug Development and Industrial Pharmacy, 1987; 13: 9-11.

4. Dhawan S, M Verma M, and Sinha VR, High Molecular weight poly (ethyleneoxide) -Based drug delivery system Part 1: Hydrogels and Hydrophilic matrix systems, Pharmaceutical Technology, 2008;12: 72-79.

5. Maggi L, Bruni R, Conte U. High molecular weight polyethylene oxides (PEOs) as an alternative to HPMC in controlled release dosage forms. International Journal of Pharmaceutics 2000; 195: 229-238.

6. Deshkar S, Pawar M, Shirsat A, Shirolkar S. Development of sustained release tablet of Mebeverine hydrochloride. Journal of Pharmaceutical Education \& Research, 2013; 4(1): 64-69.

7. Vidyadhara S, Sasidhar RLC, Nagaraju R. Design and Development of Polyethylene Oxide Based Matrix Tablets for Verapamil Hydrochloride. Indian Journal Pharm Science, 2013; 75(2):185-190.

8. Bukka R, Dwivedi M, Nargund LVG, Prasam K. Formulation and Evaluation of Felodipine Buccal Films containing Polyethylene Oxide. International Journal of Research in Pharmaceutical and Biomedical Sciences. 2012; 3 (3):1153-1158.

9. Dasbach TP and Balwinski K, Investigation of the Influence of Polyethylene Oxide in a Compression-Coated, ControlledRelease Tablet Containing a Water-Soluble Active, Poster presented at the Annual Meeting and Exposition of the American Association of Pharmaceutical Scientists Salt Lake City, Utah October 26-30, 2003. 